\title{
Selective Membrane Sensors for Free Radical Analysis Based on Potentiometric and CHEMFET Devices
}

\author{
L. Campanella, G. Favero, F. Occhionero and M. Tomassetti \\ Department of Chemistry, University of Rome "La Sapienza", Piazzale Aldo Moro 5, 00185 Roma, Italy
}

\begin{abstract}
The present paper describes a completely new method for radical determination based on spin trapping reaction suitably adapted for the electrochemical determination of radical species.

First of all benzylidenephenylnitrone was synthesized and then immobilised in a PVC-sebacate membrane. Lastly, this membrane was used to assemble different kinds of selective potentiometric sensors and CHEMFETs. These analytical devices were then used to determine free radical species, principally the anion superoxide radical and, in one case, also the hydroxide radical.
\end{abstract}

Key words. Selective sensors - CHEMFET — superoxide radical analysis.

\section{Introduction}

Despite the importance of oxygen-free radicals and their involvement in serious diseases their determination is currently performed mainly by ESR (Electronic Spin Resonance) [1], the best method, but rather expensive and difficult to apply, or else by using some specific reactions followed by spectrophotometric detection [2]. There are, for instance, comparatively few methods based on electrochemical measurements for determining free radicals. On the other hand, electrochemical sensors are generally inexpensive and easy to construct and use. In some cases they can even operate in situ. We thus recently approached the problem starting from the determination of oxygen free radicals, in particular the superoxide radical. To this end, on the one hand, we based our approach on the superoxide dismutase enzyme biosensor, which is somewhat similar to that used by E.J. Land et al. [3] and more recently by Min Ik Song et al. [4]; on the other hand, we proposed a voltammetric system based on the detection of reduced cytochrome $c$ : this system has also been applied in order to develop a suitable amperometric carbon paste electrode [5], while the third approach, i.e. the one presented in this paper, describes the main results obtained by assembling two new electrochemical sensors (one a classical selective membrane potentiometric sensor and the other a solid state glassy carbon or CHEMFET) all suitable for the determination of superoxide radicals, but, in the case of the classical sensor, also other 
free radicals (e.g. the hydroxide radical). Both these devices are based on using a selective membrane to entrap the benzylidenephenylnitrone. The benzylidenephenylnitrone selective membrane sensor is based on the peculiar properties of this substance which can generate adducts when it reacts with radicals:

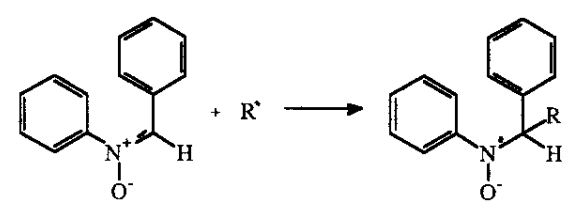

benzylidenephenylnitrone radical adduct

By means of this spin trapping reaction, starting from a less stable radical, it is possible to obtain a paramagnetic adduct that can be determined, for instance by electron spin resonance [6]. On the basis of the described possibility of obtaining an adduct of nitrones and free radicals, it is possible to prepare a nitrone-containing PVC-sebacate membrane to be used in the assembly of both radical selective potentiometric sensors (classical or glassy carbon) and a new CHEMFET device for superoxide and hydroxide radical determination.

\section{Experimental}

\section{Reagents and Apparatus}

The following reagents were used: benzylphenylamine, 2methylpiperidine, sodium tungstate bihydrate, supplied by Aldrich (Steinheim, Germany); hydrochloric acid, hydrogen peroxide, isopropyl alcohol, cyclohexanone, chloroform, dichloromethane, methanol, potassium chloride, sodium hydrate, sodium metabisulphite, anhydrous sodium sulphate, tetrahydrofuran (THF) were supplied by Carlo Erba (Milan, Italy); bis-(2-ethylhexyl)sebacate, high molecular weight polyvinylchloride (PVC), xanthine oxidase (XOD) from milk derivatives $0.39 \mathrm{U} \cdot \mathrm{mg}^{-1}$ from Fluka (Buchs, Switzerland); ferrous chloride, sodium carbonate from Merck (Darmstadt, Germany); ethylenediaminetetraacetic acid (EDTA) disodium salt, xanthine (2,6-dihydroxy purine) sodium salt supplied by Sigma (Norfolk, MO, USA).

For the nitrone electrode experimental measurements an Amel saturated calomel electrode was used as reference electrode. Both electrodes were connected to an Orion Mod. EA 940 multimeter connected to an Amel 868 analogue recorder. The instrumentation used for the CHEMFET measurements was supplied by CPL Elettronica S.r.l. (Rome, Italy).

In all cases the electrodes dipped into a $50 \mathrm{~mL}$ glass measuring cell supplied by Marbaglass (Rome, Italy) fitted with a Julabo $\mathrm{V}$ thermostat. The solution in the cell was constantly stirred using a magnetic stirrer supplied by Velp Scientifica (Milan, Italy) provided with a magnetic anchor.

The IR spectra were recorded using a IR-470 Shimadzu spectrophotometer with $\mathrm{CHCl}_{3}$ as solvent.

The NMR spectra were recorded on a Gemini $200 \mathrm{MHz}$ spectrophotometer with $\mathrm{CDCl}_{3}$ as solvent.

\section{Methods}

\section{Synthesis and characterization of benzylidenephenylnitrone}

Benzylphenylamine (a comparatively cheap and readily available product) was used as starting compound and was oxidized using H. Mitsui's procedure [7].

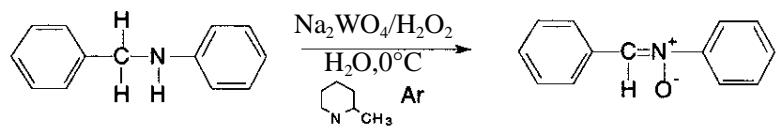

A $30 \%$ solution of $\mathrm{H}_{2} \mathrm{O}_{2}$ (5 equivalents) at $0{ }^{\circ} \mathrm{C}$ and under a flow of Ar was added to $10 \mathrm{~mL}$ of an aqueous solution of benzylphenylamine $(0.02 \mathrm{~mol})$, sodium tungstate bihydrate (0.01 mol) and 2-methylpiperidine (2.5 equivalents) placed in a two-necked flask fitted with a magnetic stirrer and rubber baffles.

The reaction mixture thus obtained was allowed to stand for $30 \mathrm{~min}$ at $0{ }^{\circ} \mathrm{C}$ under constant stirring. It was then heated to $25^{\circ} \mathrm{C}$ and maintained at this temperature for a further 30 min, again under constant stirring and in a flow of Ar.

At the end of the reaction sodium metabisulphite was added to the mixture to remove excess oxidizing agent, and then sodium chloride added until a saturated solution was obtained. The desired product was then extracted from the aqueous mixture by shaking the latter with small portions of dichloromethane. The extracts were then combined and dried over anhydrous $\mathrm{Na}_{2} \mathrm{SO}_{4}$. After filtering, the organic phase was evaporated under reduced pressure. The crude product thus obtained was purified by flash chromatography on silica gel (eluent $\mathrm{CH}_{2} \mathrm{Cl}_{2} / \mathrm{CH}_{3} \mathrm{OH}(99: 1 \mathrm{v} / \mathrm{v})$ ) to obtain the desired product with a yield of $68 \%$ by weight.

The product was characterized by comparison of the IR and NMR spectra with those of the starting amine.

IR $\left(\mathrm{CHCl}_{3}, \gamma \mathrm{cm}^{-1}\right)$ : all the characteristic peaks of the secondary starting amine, i.e. those at 3495 (stretching $\mathrm{NH}$ ), at 1600 (bending $\mathrm{NH}$ ), at 1505 (bending $\mathrm{NH}$ ) and at 1340 (stretching $\mathrm{C}-\mathrm{N}$ ) have disappeared.

$\mathrm{NMR}\left(\mathrm{CDCl}_{3}, \delta \mathrm{ppm}\right)$ : the multiplet of the starting amine at $4.20\left(\mathrm{~m}, 2 \mathrm{H}, \mathrm{CH}_{2}\right)$ referring to the $\mathrm{CH}_{2}$ in $\alpha$ position with respect to the phenyl has disappeared together with the multiplet at $3.78(\mathrm{~m}, 1 \mathrm{H}, \mathrm{NH})$ referring to the $\mathrm{NH}$; all the signals referring to the aromatic rings, namely the multiplets at $8.38(\mathrm{~m}, 2 \mathrm{H}, \mathrm{CH})$, at $7.75(\mathrm{~m}, 2 \mathrm{H}, \mathrm{CH})$, at $7.48(\mathrm{~m}, 5 \mathrm{H}, \mathrm{CH})$ and the singlet referring to $\mathrm{CH}$ : $7.91(\mathrm{~s}, 1 \mathrm{H}, \mathrm{CH})$ remain.

\section{Preparation of the PVC/sebacate selective membrane}

The PVC membrane was prepared from a polyvinylchloride solution in tetrahydrofuran (165 $\mathrm{mg}$ in $3 \mathrm{~mL}$ ) using a procedure described in a previous paper [8]. The mixture was stirred magnetically for $2 \mathrm{~h}$ at ambient temperature until complete dissolution was obtained. Then, $10 \mathrm{mg}$ of the previously synthesized nitrone, which is perfectly soluble in the solvent chosen, were added. The final product was rendered more plastic by adding to the mixture $360 \mu \mathrm{L}$ of bis(2-ethylhexyl)sebacate as plasticizer and stirring was continued. 
As soon as a homogeneous solution was obtained it was poured into a glass capsule having a diameter of $50 \mathrm{~mm}$. After $12 \mathrm{~h}$, during which the solvent was allowed to evaporate, a $0.2 \mathrm{~mm}$ thick membrane was obtained to be used to assemble the electrode.

\section{Assembly of the classical-type benzylidenephenylnitrone potentiometric sensor}

A portion of the PVC membrane containing the nitrone was glued to the end of a PVC tube (threaded at the opposite end) using PVC dissolved in cyclohexanone as adhesive. After allowing to stand for $24 \mathrm{~h}$ and having ensured that the membrane was securely glued to the cylindrical support, the PVC tube was filled with the prepared internal solution (for the superoxide radical measures: carbonate buffer $0.05 \mathrm{~mol} \mathrm{~L}^{-1}$ at $\mathrm{pH} 10.2, \mathrm{KCl} 0.01 \mathrm{~mol} \mathrm{~L}^{-1}$, xanthine oxidase $0.12 \mathrm{mg} \mathrm{mL}^{-1}$ and xanthine $0.01 \mathrm{~mol} \mathrm{~L}^{-1}$; while for the hydroxide radical measures: EDTA $2.0 \times 10^{-2} \mathrm{~mol} \mathrm{~L}-1, \mathrm{FeCl}_{2}$ $2.0 \times 10^{-2}$ mol L${ }^{-1}$ at $\mathrm{pH} 5.0$ and $\mathrm{H}_{2} \mathrm{O}_{2} 0.01 \mathrm{~mol} \mathrm{~L}^{-1}$ ) and was screwed to a support housing an $\mathrm{Ag} / \mathrm{AgCl}$ inner reference electrode, as shown in figure 1a.

\section{Assembly of glassy carbon benzylidenephenylnitrone potentiometric sensor}

The nitrone sensor was also assembled as a solid state sensor: as shown in figure $1 \mathrm{~b}$, the head of a glassy carbon electrode was covered with a selective polymeric membrane obtained by depositing $50 \mu \mathrm{L}$ of a tetrahydrofuran solution $(1.5 \mathrm{~mL})$ containing $30 \mathrm{mg}$ of PVC base polymer, $66 \mathrm{mg}$ bis(2-ethylhexyl)sebacate and $4 \mathrm{mg}$ ( $4 \%$ by weight) of benzylidenephenylnitrone and allowing the solvent to evaporate completely at ambient temperature for $24 \mathrm{~h}$.

\section{Assembly of the benzylidenephenylnitrone CHEMFET}

A second type of solid state sensor was obtained using a field effect transistor (FET) as transducer.

For this purpose [9] an integrated chip was used (UUO3 type, supplied by the HEDCO Laboratory of Utah University (USA)). This chip (overall dimensions $1.28 \mathrm{~mm} \times 2.16 \mathrm{~mm}$ ) contained two $400 \mu \mathrm{m} \times 20 \mu \mathrm{m}$ gates and two metal gate control devices. The chip was accurately washed before use with isopropyl alcohol and then glued using an epoxy resin to a plastic stick, which was subsequently connected to the electrical measurement system. After making the electrical connections with an ultrasonic wire-bonder (Kulicke and Soffa, model 4123, Switzerland), the chip was encapsulated in an epoxy resin (EPON 825+ Jeffamine D-230) body, leaving free only the area of the two gates. The FET gate was then covered with the polymeric membrane made of polyvinylchloride (PVC) sebacate and containing benzylidenephenylnitrone. This was done by stirring for about $5 \mathrm{~h}$ a suspension consisting of $30 \mathrm{mg}$ of PVC as base polymer, $66 \mathrm{mg}$ di bis(2-ethylhexyl)sebacate as plasticizer and $4 \mathrm{mg}$ ( $4 \%$ by weight) of benzylidenephenylnitrone in $1.5 \mathrm{~mL}$ of tetrahydrofuran. The solvent was allowed to evaporate partially in order to obtain a sufficiently viscous suspension. A drop (about $50 \mu \mathrm{L}$ ) of this suspension was deposited on the FET gate area, taking care to avoid air bubble formation, and left to dry at room temperature for $24 \mathrm{~h}$. (a)

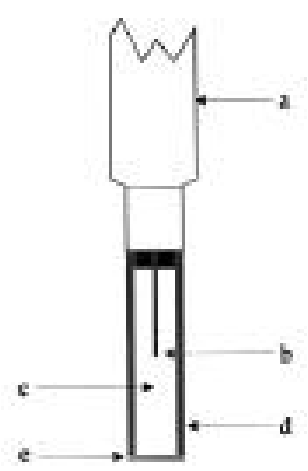

(b)

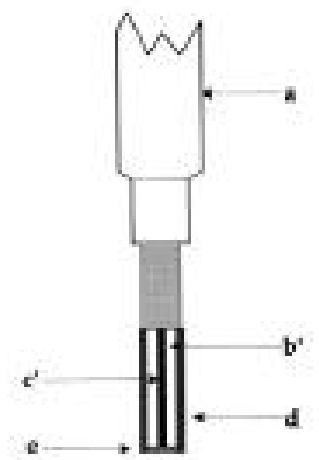

a: electrode plastic body; b: inner reference $\mathrm{Ag} / \mathrm{AgCl} / \mathrm{Cl}$ - electrode; $\mathbf{b}^{\prime}$ : glassy carbon wire; c: inner solution; $\mathbf{c}^{\prime}$ : teflon body; $\mathbf{d}$ : PVC tube; e: PVC membrane containing the benzylidenephenylnitrone.

Fig. 1. Selective potentiometric membrane electrodes assembly, for the determination of free radicals based on a PVC/sebacate/benzylidenephenilnitrone selective membrane: (a): classical sensor, (b): glassy carbon electrode.

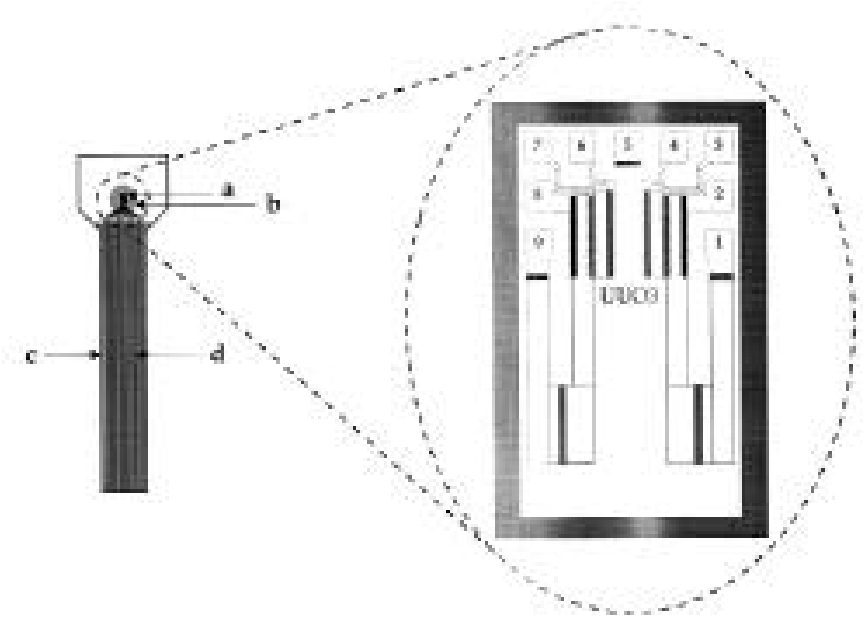

a: FET; b: polymeric membrane containing benzylidenephenylnitrone; c: plastic stick; d: electric wires; 1,2: ISFET 1 source and drain; 3: MOSFET 1 gate; 2,4: MOSFET 1 source and drain; 5: silicon substrate; 6,8: MOSFET 2 source and drain; 7: MOSFET 2 gate; 8,9: ISFET 2 source and drain.

Fig. 2. CHEMFET assembly, for the determination of free radicals based on a FET as transducer and a PVC/sebacate/benzylidenephenil-nitrone selective membrane. ure 2 .

The assembly of the solid state sensor is illustrated in fig-

\section{Performance of measures using nitrone sensors}

The sensor (classical selective membrane type, or solid state (glassy carbon or FET)), assembled as previously described, was dipped, together with a satur ated calomel reference electrode, in the measuring cell fitted with a glass jacket and thermostatted at $25{ }^{\circ} \mathrm{C}$, containing $25 \mathrm{~mL}$ of the measurement solution (which, in the case of superoxide radical measures, consisted of carbonate buffer $0.05 \mathrm{~mol} \mathrm{~L}^{-1} \mathrm{pH} 10.2$, 
$\mathrm{KCl} 0.01 \mathrm{~mol} \mathrm{~L}^{-1}$, EDTA $1 \times 10^{-4} \mathrm{~mol} \mathrm{~L}^{-1}$ and xanthine oxidase $0.12 \mathrm{mg} \mathrm{mL}^{-1}$, while, in the case of hydroxide radical measures it consisted of EDTA $2.0 \times 10^{-2} \mathrm{~mol} \mathrm{~L}-1, \mathrm{FeCl}_{2}$ $2.0 \times 10^{-2} \mathrm{~mol} \mathrm{~L}^{-1}$ at $\mathrm{pH} 5.0$ ): both sensor and reference electrode were connected to a potentiometer from which the membrane potential could be read off, in the case the sensor was of the classical potentiometric or glassy carbon type, while the output voltage potential was recorded on special measuring apparatus, as described in preceding papers [9] in the case of a FET-based solid state sensor. In all cases the variations in potential before and after addition of reagent able to develop the free radical in situ were recorded as described in the following section.

\section{Generation in situ of superoxide radicals}

One classical method for generating superoxide radicals in the aqueous phase exploits the reaction of xanthine oxidation by xanthine oxidase $[10,11]$ :

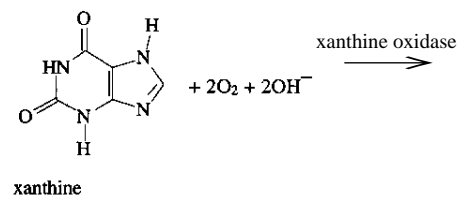<smiles>O=c1[nH]c(=O)c2c([nH]1)[nH]c1[nH]c(=O)[nH]c(=O)c12</smiles>

In the experiments described in the present paper, the superoxide radical species was generated in situ in the aqueous solution of carbonate buffer $0.05 \mathrm{~mol} \mathrm{~L}^{-1}$, EDTA $1 \times 10^{-4} \mathrm{~mol} \mathrm{~L}^{-1}$, pH 10.2 containing $0.12 \mathrm{mg} \mathrm{mL}^{-1}$ of xanthine oxidase.

\section{Generation in situ of hydroxide radicals}

Hydroxide radicals can be generated in situ in aqueous solvent using a procedure known as "Fenton's reagent" $[2,12]$ : the latter consists in the reaction of $\mathrm{H}_{2} \mathrm{O}_{2}$ with an equimolar solution of ferrous ion and EDTA which produces hydroxide radicals according to the following reaction:

$$
\mathrm{Fe}^{2+}+\mathrm{H}_{2} \mathrm{O}_{2} \rightarrow \mathrm{Fe}^{3+}+\mathrm{HO}^{-}+\mathrm{HO}^{-}
$$

The operating conditions used thus consisted of an aqueous solution at $\mathrm{pH} 5.0$ in the presence of $\mathrm{Fe}(\mathrm{II})$ ion $2.0 \times$ $10^{-2} \mathrm{~mol} \mathrm{~L}^{-1}$ and EDTA $2.0 \times 10^{-2} \mathrm{~mol} \mathrm{~L}^{-1}$ adding quantities of $\mathrm{H}_{2} \mathrm{O}_{2}$ such as to obtain hydrogen peroxide concentrations in the solution in the range of $10^{-4}$ to $10^{-2} \mathrm{~mol} \mathrm{~L}^{-1}$.

\section{Results}

As described previously, the selective membrane sensor containing the benzylidenephenylnitrone was based on the peculiar property of this type of substance (nitrones) to produce reaction adducts with free radicals through the socalled spin trapping reaction.

It is thus necessary to prepare a membrane containing a nitrone, which it had been possible to use to build a radicalselective potentiometric sensor.

It was initially hypothesized that the substance capable of endowing the membrane with selectivity towards radical species might be one of those already used in the spin trapping technique, that is, one of the substances used in spin electronic resonance to stabilize the radical, i.e. nitrones or
$\mathrm{N}$-oxides available also on the market. In fact several commercially available nitrones and N-oxides are commonly used to determine radicals by means of spin electronic resonance, although they are species that are highly soluble in aqueous solution. This represented a barrier to the direct use of one of the commercial species as, in order to dissolve it in a PVC and sebacate membrane, it would be necessary to use a nitrone that was not too hydrophilic.

The procedure adopted was therefore to synthesize a suitable product having the characteristics described above and use it to build the selective membrane of the electrode. Of course, any such compound would have to have at least one lipophilic group (single or condensed aromatic rings, alkyl chains, etc.) to make it comparatively insoluble in water.

Benzylidenephenylnitrone was thus synthesized for this purpose (see reaction (1)).

After synthesizing the required nitrone and immobilizing it in the PVC membrane and using the latter to build a classical potentiometric membrane sensor, as described in the Experimental section, the analytical device was used to determine two different radical species: the superoxide anion radical and the hydroxide radical.

The results for the first of these two radical species are shown graphically in figure 3 , in which sensor response (in $\mathrm{mV}$ ) is plotted against the logarithm of the concentration of the added species, while the main analytical results obtained are set out in table I. As can be seen, the system displays a good response linearity over a range of about one decade.

To confirm that the system was functioning regularly blank tests were then carried out by assembling the sensor using a PVC and sebacate membrane not containing the benzylidenephenylnitrone species required for determining the radicals. The results of the blank test are compared with those of the preceding tests in figure 3. It is apparent how, in the absence of the nitrone species, as there is no way of forming the adduct between the latter and the radical

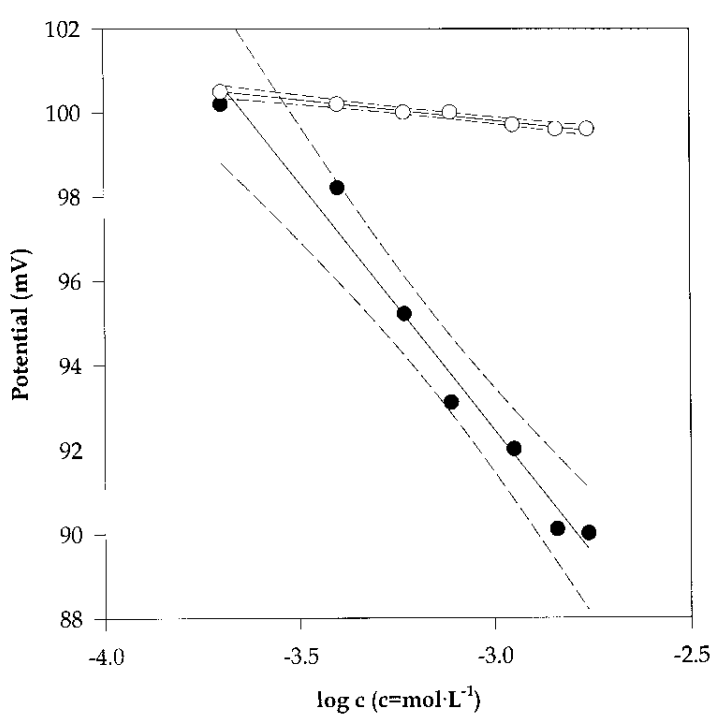

Fig. 3. Calibration curve for superoxide radical obtained by using the classical selective membrane potentiometric sensor (-) response of the sensor; $O$ : blank). Equation of the calibration curve and confidence interval $\left(y=\mathrm{mV} ; x=\mathrm{mol} \mathrm{L} \mathrm{L}^{-1}\right)$ : $y=-11.8( \pm 2.4) \cdot \log x+57.2( \pm 6.2)$. 
Table I. Analytical characterisation of classical selective membrane potentiometric sensor for the determination of superoxide and hydroxide radicals.

\begin{tabular}{lcc}
\hline & $\begin{array}{c}\text { Superoxide } \\
\text { radical }\end{array}$ & $\begin{array}{c}\text { Hydroxide } \\
\text { radical }\end{array}$ \\
\hline Linearity range (mmol L-1) & $0.20-1.7$ & $0.30-1.8$ \\
Low detection limit $\left(\mathrm{mmol} \mathrm{L}^{-1}\right)$ & 0.03 & 0.03 \\
Precision (as \% RSD) & 8.8 & 9.1 \\
Response time (s) & $\leq 60$ & $\leq 60$ \\
Lifetime (days) & $\sim 5$ & $\sim 5$ \\
\hline
\end{tabular}

Table II. Analytical characterisation of solid state selective membrane devices for the determination of superoxide radical: (A) using the glassy carbon potentiometric sensor and (B) using the CHEMFET device.

\begin{tabular}{lcc}
\hline & $\begin{array}{c}(A) \\
\text { Superoxide } \\
\text { radical }\end{array}$ & $\begin{array}{c}(B) \\
\text { Superoxide } \\
\text { radical }\end{array}$ \\
\hline Linearity range (mmol L-1) & $0.10-1.9$ & $0.025-1.0$ \\
Low detection limit (mmol L-1 & 0.03 & 0.01 \\
Precision (as \% RSD) & 9.8 & 10.0 \\
Response time (s) & $\leq 30$ & $\leq 20$ \\
Lifetime (days) & $\sim 2$ & $\sim 1$ \\
\hline
\end{tabular}

species, no variation is observed in the signal emitted by the system that can be related to the xanthine additions, while only a slight drift in the signal itself is recorded.

As further confirmation of the sensor's capacity to respond to radical species the determination of a different radical species, namely the hydroxide radical, was performed. This radical was generated in solution by addition of $\mathrm{H}_{2} \mathrm{O}_{2}$ (Fenton's reagent) as described in the Experimental section. Also in this case (see Fig. 4) variations were observed in the sensor signal corresponding to the additions to the solution of amounts of the species capable of generating the radical and the main analytical data are summarized in table I. Of course, also in this case a blank test was run. It was found (Fig. 4) that in the absence of nitrone there were no appreciable variations in the signal.

Also in the case of the hydroxide radical the system's response was found to be linear over a concentration range of about one decade. Sensitivity was apparently greater towards the hydroxide radical than towards the superoxide radical, as the value of the slope of the linear section of the calibration curve was about double that of the superoxide radical. The low detection limit, calculated as the concentration corresponding to three times the background noise, was in both cases about $0.03 \mathrm{mmol} \mathrm{L}^{-1}$.

In view of the ease of their construction and the extreme flexibility demonstrated by the solid state selective electrodes of the glassy carbon type built in recent years by our research group $[13,14]$, we deemed it advisable to attempt construction also in this case.

The principal analytical data obtained by determining the superoxide radical using the glassy carbon benzylidenephenylnitrone sensor are shown in table II, while the calibration curve and the related blank test curve are shown in figure 5.

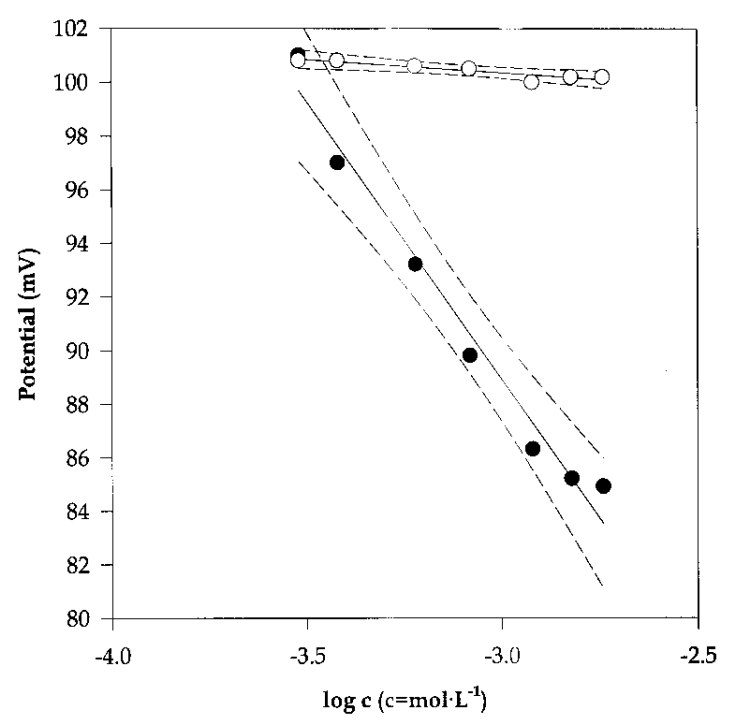

Fig. 4. Calibration curve for hydroxide radical obtained by using the classical selective membrane potentiometric sensor (०: response of the sensor; $O$ : blank). Equation of the calibration curve and confidence interval $\left(y=\mathrm{mV} ; x=\mathrm{mol} \mathrm{L} \mathrm{L}^{-1}\right)$ : $y=-20.7( \pm 3.7) \cdot \log x+26.7( \pm 9.3)$.

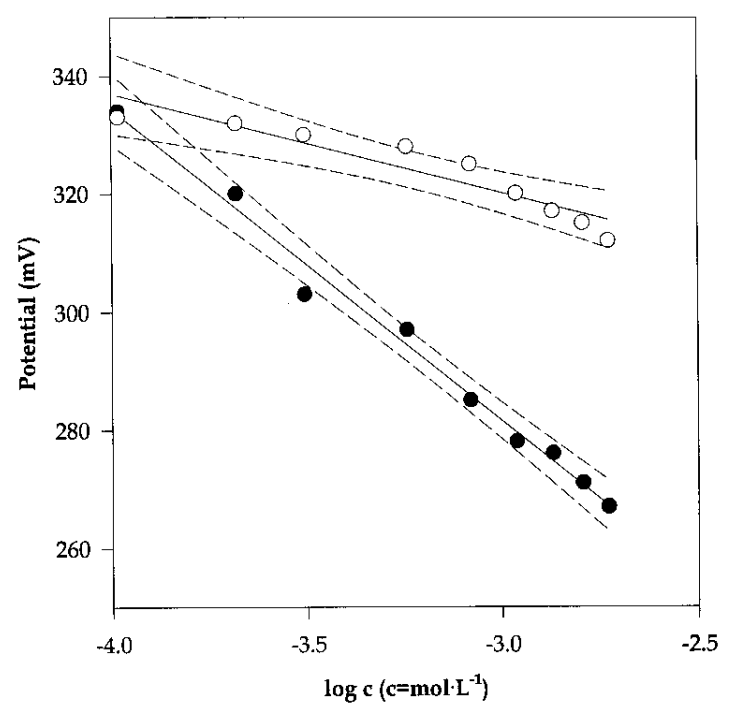

Fig. 5. Calibration curve for superoxide radical obtained by using the selective membrane glassy carbon potentiometric sensor. (O: response of the sensor; $O$ : blank). Equation of the calibration curve and confidence interval $\left(y=\mathrm{mV} ; x=\mathrm{mol} \mathrm{L}^{-1}\right)$ : $y=-52.8( \pm 5.9) \cdot \log x+123.0( \pm 14.3)$.

The results obtained so far using a modified FET based on a benzylidenephenylnitrone-based membrane are also summarised in table II and shown in figure 6. Despite the low stability, it can be seen that, even at the present state of the art of the device, it is possible to record a signal that correlates reasonably well with the concentration of the superoxide radical produced in solution by the xanthine additions. 


\section{Original articles}

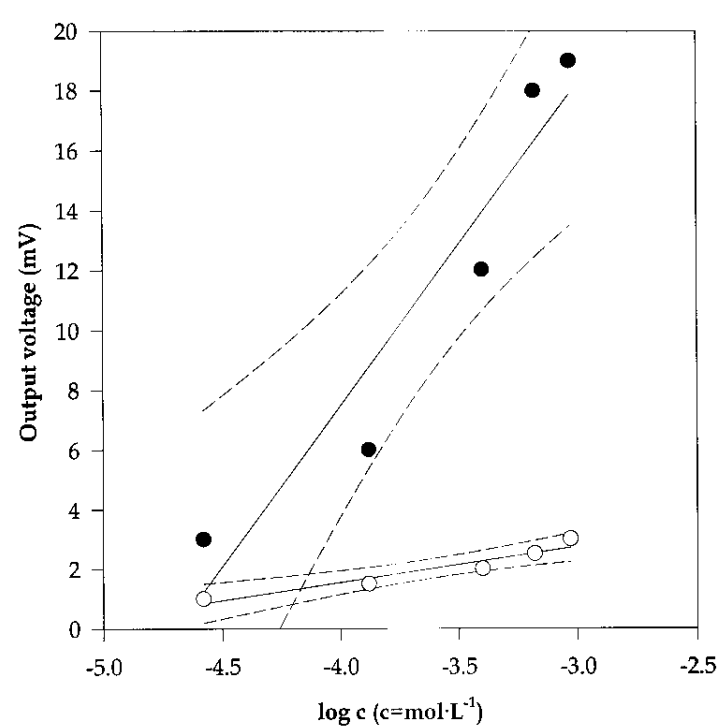

Fig. 6. Calibration curve for superoxide radical obtained by using the CHEMFET device ( $\bullet$ : response of the sensor; $\bigcirc$ : blank). Equation of the calibration curve and confidence interval $(y=\mathrm{mV}$; $x=$ mol L $\left.{ }^{-1}\right): y=10.7( \pm 4.7) \cdot \log x+50.5( \pm 14.6)$.

As far as the devices developed are concerned, from the analytical point of view, the classical potentiometric selective membrane sensor gives a linear response in the range $0.2-1.7 \mathrm{mmol} \mathrm{L}^{-1}$ for superoxide radicals and in the range $0.3-1.8 \mathrm{mmol} \mathrm{L}^{-1}$ for hydroxide radicals. The solid state glassy carbon electrode gives an analogous response between $0.1-1.9 \mathrm{mmol} \mathrm{L}^{-1}$ for the superoxide radical, while the sensor based on the field effect transistor, gives a linear response in the range $25 \mu \mathrm{mol} \mathrm{L}-1-1.0 \mathrm{mmol} \mathrm{L}^{-1}$ with for the same radical. The possible functioning of the latter type of sensor has been tested since they are easy to miniaturize. However, several problems of stability remain to be solved which seriously jeopardise the repeatability of the response. Another current problem with this type of sensor is the short working life of the nitrone-containing membranes (see data in the respective tables). This seems to be largely due to a kind of "saturation" effect in the membrane due to the spin trapping reaction.

\section{Conclusions}

This research is a basic work in view of the small number of sensors for radicals reported in literature. One of the most interesting features of the work is, in our opinion, the preparation of the species lipophilic and capable of forming the adduct with the radicals, namely the benzylidenephenylnitrone. The synthesis of a lipophilic species that, as far as interaction with the radicals is concerned, behaves like the commercially available hydrophilic species, and the testing of its use in a selective polymeric membrane for the purpose of radical determination is without doubt a step forward in this research sector that will hopefully produce new analytical devices in future that are capable of providing not only an integral response for radical concentration, but probably also selective responses, provided that it is possible to prepare molecules that can ensure a spin trapping reaction using only one type of free radical.

In conclusion, although the above-mentioned problems need further investigation, the results obtained in the present work provide a useful starting point for further research in this field.

\section{Acknowledgement}

The authors wish to thank the National Research Council (CNR) of Italy, Target Projects in "Solid State Electronic Materials" (MADESS) and "Tecnologie Chimiche Innovative" for the financial support.

\section{References}

1. Carey, F. A.; Sundberg, R. J. "Advanced Organic Chemistry (second edition) ", Plenum Press, New York, 1984.

2. Naughton, D. P.; Grootveld, M.; Blake, D. R.; Guestrin, H. R.; Narayanaswamy, R. Biosens. Bioelectr. 1993, 8, 325-329.

3. Land, E. J.; Swallow, A. J. Arch. Biochem. Biophys. 1971, 145, 365-372.

4. Song, M. I.; Bier, F. F.; Scheller, F. W. Bioelectrochem. Bioenerg. 1995, 38, 419-422.

5. Campanella, L.; Favero, G.; Tomassetti, M. Sensors \& Actuators B 1997, 44, 559-565.

6. Mason, R. P.; Knecht, K. T. Methods in Enzymology 1994, 233, 112-117.

7. Mitsui, H.; Zenki, S.; Shiota, T.; Murahashi, S. J. Chem. Soc., Chem. Commun. 1984, 13, 874-875.

8. Campanella, L.; Aiello, L.; Colapicchioni, C.; Tomassetti, M. Anal. Lett. 1997, 30, 1611-1629.

9. Campanella, L.; Tomassetti, M.; Su, Y.; Crescentini, G.; Sammartino, M. P. Chem. today 1996, march/april, 33-40.

10. Fridovich, I. J. Biol. Chem. 1970, 245, 4053-4057.

11. Beauchamp, C.; Fridovich, I. Anal. Biochem. 1971, 44, 276287.

12. Walling, C.; Johnson, R. J. Am. Chem. Soc. 1975, 97, 363-367.

13. Campanella, L.; Su, Y.; Crescentini, G.; Sammartino, M. P.; Tomassetti, M. Anal. Lett. 1994, 27, 429-452.

14. Campanella, L.; Mazzei, F.; Sbrilli, R.; Tomassetti, M. Ann. Chim. 1990, 80, 395-412. 